\title{
Accuracy of ECG-based screening for sleep-disordered breathing: a survey of all male workers in a transport company
}

\author{
Junichiro Hayano • Teruomi Tsukahara • \\ Eiichi Watanabe · Fumihiko Sasaki • Kiyohiro Kawai • \\ Hiroki Sakakibara • Itsuo Kodama • \\ Tetsuo Nomiyama $\cdot$ Keisaku Fujimoto
}

Received: 6 January 2012 /Revised: 8 February 2012 / Accepted: 5 March 2012 /Published online: 20 March 2012

(C) The Author(s) 2012. This article is published with open access at Springerlink.com

\begin{abstract}
Purpose Sleep-disordered breathing (SDB) is associated with increased risk for cardiovascular morbidity and mortality and for sleepiness-related accidents, but $>75 \%$ of the patients remain undiagnosed. We sought to determine the diagnostic accuracy of ECG-based detection of SDB when used for population-based screening.

Methods All male workers, mostly truck drivers, of a transport company ( $n=165$; age, $43 \pm 12$ years) underwent standard attended overnight polysomnography. Cyclic variation of heart rate (CVHR), a characteristic pattern of heart rate associated with SDB, was detected from single-lead ECG signals during the polysomnography by a newly developed automated algorithm of autocorrelated wave detection with adaptive threshold (ACAT).
\end{abstract}

J. Hayano $(\bowtie)$

Center for Medical Education Research and Development,

Nagoya City University Graduate School of Medical Sciences,

1 Kawasumi Mizuho-cho Mizuho-ku,

Nagoya, Aichi 467-8601, Japan

e-mail: hayano@med.nagoya-cu.ac.jp

T. Tsukahara $\cdot$ T. Nomiyama

Department of Preventive Medicine and Public Health,

Shinshu University School of Medicine,

3-1-1 Asahi, Matsumoto,

Nagano 390-8621, Japan

E. Watanabe

Division of Cardiology, Department of Internal Medicine,

Fujita Health University School of Medicine,

1-98 Dengakugakubo, Kutsukake-cho,

Toyoake, Aichi 470-1192, Japan
Results Among 165 subjects, the apnea-hypopnea index (AHI) was $\geq 5$ in $62(38 \%), \geq 15$ in $26(16 \%)$, and $\geq 30$ in $16(10 \%)$. The number of CVHR per hour (CVHR index) closely correlated with AHI $[r=0.868(95 \%$ CI, $0.825-$ $0.901)]$. The areas under the receiver operating characteristic curves for detecting subjects with $\mathrm{AHI} \geq 5, \geq 15$, and $\geq 30$ were 0.796 (95\% CI, 0.727-0.855), 0.974 (0.937-0.993), and 0.997 (0.971-0.999), respectively. With a predetermined criterion of CVHR index $\geq 15$, subjects with AHI $\geq 15$ were identified with $88 \%$ sensitivity and $97 \%$ specificity (likelihood ratios for positive and negative test, 30.7 and 0.12 ). The classification performance was retained in subgroups of subjects with obesity, hypertension, diabetes mellitus, dyslipidemia, and decreased autonomic function.

F. Sasaki $\cdot$ H. Sakakibara

Takaoka Clinic Nagoya,

2-28-24 Izumi, Higashi-ku,

Nagoya, Aichi 461-0001, Japan

K. Kawai

Suzuken Company Limited,

8 Higasikataha, Higashi-ku,

Nagoya, Aichi 461-0015, Japan

I. Kodama

Nagoya University,

Furo-cho, Chikusa-ku,

Nagoya, Aichi 464-8601, Japan

K. Fujimoto

Department of Biomedical Laboratory Sciences,

Shinshu University School of Health Sciences,

3-1-1 Asahi, Matsumoto,

Nagano 390-8621, Japan 
Conclusions The CVHR obtained by the ACAT algorithm may provide a useful marker for screening for moderate-tosevere SDB among apparently healthy male workers.

Keywords Apnea-hypopnea index - Cyclic variation of heart rate $\cdot$ Electrocardiogram $\cdot$ Sleep apnea $\cdot$ Sleep-disordered breathing $\cdot$ Population

\section{Introduction}

Sleep-disordered breathing (SDB) is a contemporary challenge to health and well-being [1-3]. Studies have demonstrated that SDB increases the risk of hypertension [4], coronary artery disease [5, 6], stroke [7], diabetes [8], chronic kidney disease [9], depression [10], cognitive impairment, diminished quality of life [11], and motor vehicle crashes [12]. Despite these facts and the availability of effective treatments, at least $75 \%$ of the patients with SDB remain undiagnosed [13]. Establishing efficient medical and public health system for SDB screening is therefore an urgent concern.

ECG seems potentially the most practical tool for screening for SDB. Episodes of SDB are accompanied by a characteristic pattern of heart rate, known as cyclic variation of heart rate (CVHR) [14], which consists of bradycardia during apnea followed by rapid return with its cessation. Earlier studies have reported several ECG-based algorithms for detecting CVHR that demonstrate good classification performance between SDB patients and normal subjects [15-18]. These studies, however, were based on observations either in small test data $[15,16]$ or in subjects referred for polysomnography with suspected SDB [18-20]. The classification performance depends on pretest probability [21] and comorbidities that are known to affect CVHR [14, 22].

In the present study, we therefore sought to determine the diagnostic accuracy of ECG-based SDB detection in population-based screening. We used a newly developed automated algorithm of autocorrelated wave detection with adaptive threshold (ACAT) for detecting CVHR [19]. This algorithm has been reported to identify patients with moderate-to-severe SDB with $83 \%$ sensitivity and $88 \%$ specificity among 862 patients referred for diagnostic polysomnography [19]. We performed overnight polysomnography in all male workers in a transport company and examined the classification performance of the algorithm.

\section{Methods}

Participants

All male workers of a transport company were eligible for inclusion in this study. Most of them were involved in driving trucks for long distances. All male workers gave their written informed consent to participate in this study. Consequently, we performed a complete survey of all male workers $(n=165)$ of this company. All subjects were racially Japanese.

Protocol

The protocol of this study was approved by the Human Ethics Committee of Shinshu University School of Medicine (No. 658, January 4, 2006). Between February 2006 and August 2007, subjects underwent polysomnography as well as a medical checkup for medical history, physical examination, blood sampling, and daytime sleepiness by the Epworth Sleepiness Scale.

Sleep study

Standard overnight attended polysomnography was performed in a university laboratory starting at 20:00 h, and the data were collected from 21:00 $\mathrm{h}$ to $06: 00 \mathrm{~h}$ the next morning. The bed was covered with a sheet-form respiratory movement sensor for the purpose of another study [23]. The polysomnogram was recorded with a digital polygraph (Alice III; Chest Co. Ltd., Tokyo, Japan). We used the standard polysomnographic montages consisting of C4-A2, C3-A1, O2-A1, and O1-A2 electroencephalograms, left and right electrooculograms, a submental electromyogram, a nasal pressure cannula, oronasal airflows, left and right tibial electromyograms, thoracoabdominal inductance plethysmograms, pulse oximetric arterial blood oxygen saturation $\left(\mathrm{SpO}_{2}\right)$, a neck microphone, body position sensors, and a modified lead II ECG.

The sleep stages and respiratory events were scored according to the AASM Manual for the Scoring of Sleep and Associated Events [24]. The average hourly frequency of apneic and hypopneic episodes was defined as apneahypopnea index (AHI). Total sleep time (TST) was used as the denominator in the calculation of AHI. Subjects with AHI between 5 and 15 were defined as mild SDB, those with AHI between 15 and 30 as moderate SDB, and those with $\mathrm{AHI} \geq 30$ as severe SDB.

\section{Detection of CVHR}

The modified lead II ECG signal (sampling frequency, $100 \mathrm{~Hz}$ ) for the entire length of each polysomnogram was extracted. All QRS complexes were identified and labeled as normal (sinus rhythm), ventricular ectopic, supraventricular ectopic, and artifact, and R-R interval time series were generated using only normal-to-normal intervals. The detection of CVHR was performed by a technician who was blind to both subject characteristics and other polysomnographic 
findings. Using the automated ACAT algorithm [19], CVHR was detected as the cyclic occurrence of autocorrelated waves (Fig. 1). See Appendix for the detail of the ACAT algorithm.

We calculated CVHR index as the average number of CVHRs (dips meeting the criteria) per hour of time in bed (TIB). We used CVHR index $\geq 15$ that had been determined by a previous study [19] as the criterion for identifying patients with moderate-to-severe SDB. We also calculated standard deviation of normal-to-normal $\mathrm{R}-\mathrm{R}$ intervals (SDNN) as an index of cardiac autonomic function and defined subjects with a SDNN $<75$ ms (mean minus $1 \mathrm{SD}$ ) as having decreased autonomic function.

\section{Statistical analysis}

The correlation and agreement between the CVHR index and AHI were evaluated by Pearson's product moment correlation coefficient and the limits of agreement of Bland and Altman [25], respectively. The classification performance of the CVHR index was evaluated by receiver operating characteristic (ROC) curve analysis. The difference in the performance was evaluated by comparing the areas under the curve (AUC) by the method of Hanley and McNeil [26]. The guideline of the American College of Chest Physicians and other related academic societies [27] recommends the use of likelihood ratios (LRs) for assessing the utility of diagnostic alternatives to polysomnography; it also offers multiple threshold approaches, one for best reducing the probability (false negative rate) and the other for best increasing the probability (true positive rate), allowing a gray zone, where the result of screening test is accepted as indeterminate. Accordingly, we calculated LRs for positive test $(\mathrm{LR}+)$ and negative test (LR-) to evaluate the classification performance and also performed interval LR analysis to determine the thresholds for increasing and reducing the post-test probabilities. We assessed the changes in disease probability with the levels of LR as follows: $<0.05$, very large reduction; $0.05-0.1$, large reduction; $0.1-0.2$, modest reduction; 0.21-5.0, little change; 5.1-10.0, modest increase; 10.1-20.0, large increase; and $>20.0$, very large increase. Data are presented as mean \pm SD (range) when appropriate. We defined a $P$ value $<0.05$ as statistically significant.
Fig. 1 a A strip of polysomnographic data with the markers of cyclic variation of heart rate (CVHR) detected by the autocorrelated wave detection with adaptive threshold (ACAT) algorithm in a representative subject with sleep-disordered breathing (SDB). The temporal positions of detected CVHR are indicated with short vertical bars. b Closer view of the data in the open box in (a). The ACAT algorithm detected the nadirs of cyclic dips in interbeat intervals that accompany apnea-hypopnea events. RRI interbeat interval measure as $\mathrm{R}-\mathrm{R}$ interval of ECG, Resp respiration by oronasal airflow, $\mathrm{SpO}_{2}$ pulse oximetric arterial blood oxygen saturation

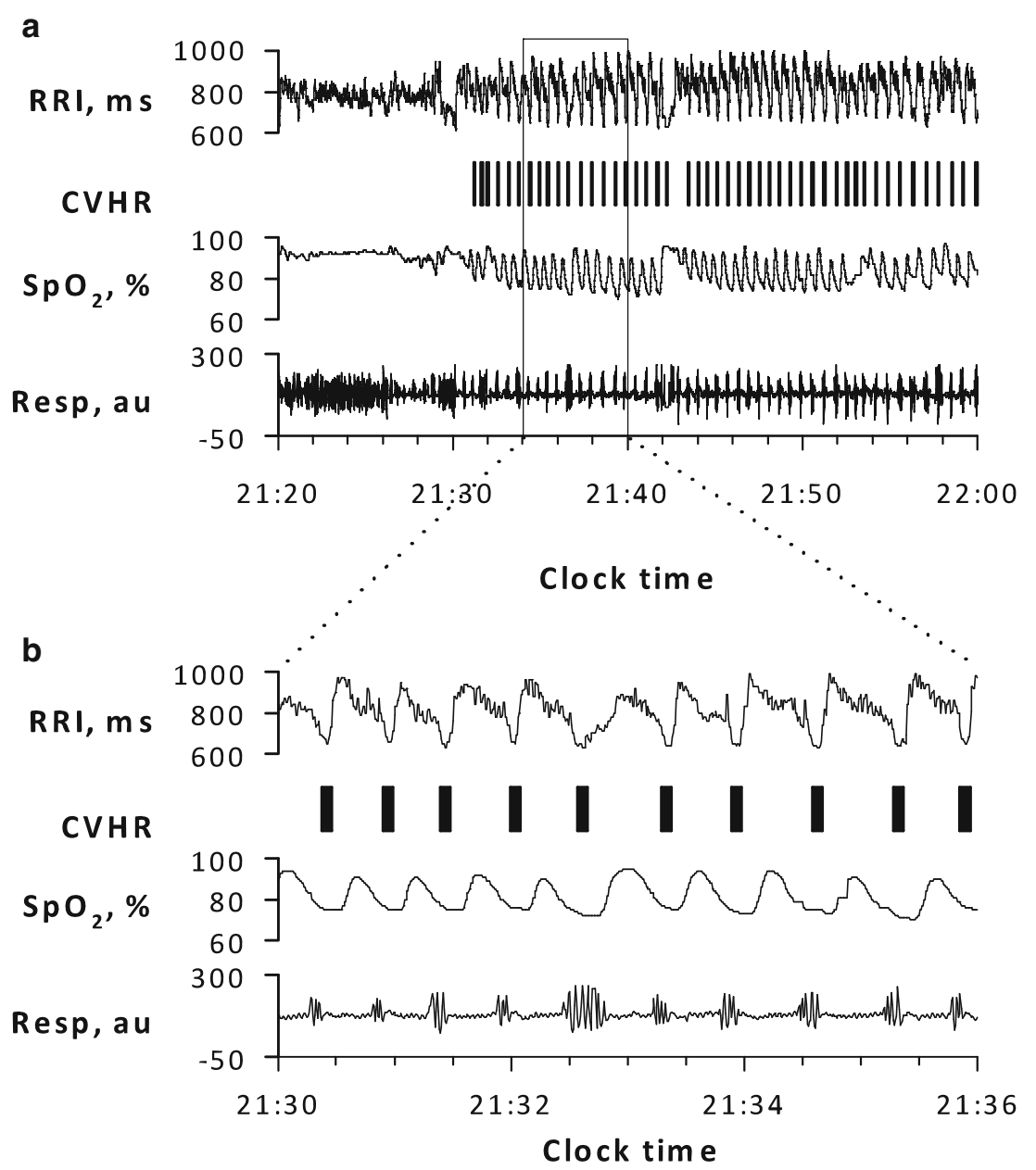




\section{Results}

Table 1 summarizes the characteristics of the subjects. They included 57 (35\%) patients with hypertension, 13 (8\%) with diabetes mellitus, and 83 (50\%) with dyslipidemia. The polysomnography revealed that the sleep efficiency was $78 \pm 13 \%$ and that the median (interquartile rage) of AHI was 3.1 (0.9-8.6). Out of 165 subjects, $62(38 \%)$ had AHI $\geq 5$, including 36 (22\%) with mild, 10 (6\%) with moderate, and 16 (10\%) with severe SDB.

The ECG in all 165 subjects showed sinus rhythm. The ratio of analyzable $\mathrm{R}-\mathrm{R}$ intervals to the recording length was $94 \pm 9 \%(44-100 \%)$. Although this ratio was $<70 \%$ only in four subjects due to recording noises, these subjects were also included in the analysis (intension-to-diagnose policy). Both the AHI and the CVHR index were $<15$ in all of these

Table 1 Characteristics of the study subjects

\begin{tabular}{ll}
\hline$N$ & 165 \\
\hline Age, years & $43 \pm 12(18-69)$ \\
Height, cm & $170 \pm 6(155-186)$ \\
Weight, $\mathrm{kg}$ & $68 \pm 11(49-112)$ \\
BMI, $\mathrm{kg} / \mathrm{m}^{2}$ & $24 \pm 3(16-37)$ \\
BMI $\geq 25 \mathrm{~kg} / \mathrm{m}^{2}$ & $50(30 \%)$ \\
BMI $\geq 35 \mathrm{~kg} / \mathrm{m}^{2}$ & $1(1 \%)$ \\
Systolic blood pressure, mmHg & $126 \pm 18(96-186)$ \\
Diastolic blood pressure, $\mathrm{mmHg}$ & $81 \pm 12(56-118)$ \\
ESS score & $5 \pm 4(0-19)$ \\
ESS score $>11$ & $11(7 \%)$ \\
ESS score $>16$ & $3(2 \%)$ \\
Comorbidity & \\
Hypertension, $n(\%)$ & $57(35 \%)$ \\
Diabetes mellitus, $n$ (\%) & $13(8 \%)$ \\
Dyslipidemia, $n$ (\%) & $83(50 \%)$ \\
Polysomnographic features & \\
TIB, min & $623 \pm 87(276-770)$ \\
TST, min & $485 \pm 104(125-683)$ \\
Rapid-eye-movement period, min & $82 \pm 35(8-180)$ \\
Sleep efficiency, \% & $78 \pm 13(27-98)$ \\
AHI (TIB), events/h & $8 \pm 13(0-63)$ \\
AHI (TST), events/h & $9 \pm 14(0-67)$ \\
Maximum O 2 desaturation, $\%$ & $9 \pm 6(0-35)$ \\
SDNN, ms & $105 \pm 32(44-179)$ \\
\hline & \\
\hline
\end{tabular}

Data are shown as number (\%) for categorical variables and mean \pm SD (range) for continuous variables

$A H I$ apnea-hypopnea index, BMI body mass index, ESS Epworth Sleepiness Scale, $S D N N$ standard deviation of normal-to-normal R-R interval, TIB time in bed, TST total sleep time subjects but one, in whom the ratio of analyzable $\mathrm{R}-\mathrm{R}$ intervals was only $44 \%$ and the CVHR index and AHI were 17.6 and 35.8 , respectively.

Figure 2 shows the relationship and agreement between the CVHR index and AHI. The CVHR index was correlated with AHI $[r=0.868(95 \%$ CI, 0.825-0.901)]. The Bland and Altman plot showed upper and lower limits of agreement of 13.1 and -16.1 , respectively, with a tendency of increasing difference with increasing AHI. The ROC curve analysis for identification of patients with moderate-to-severe SDB showed an AUC of 0.974 (SE, 0.0142; $P<0.0001$ ) and by the criterion of CVHR index $\geq 15$, the patients were detected at $88 \%$ sensitivity and $97 \%$ specificity (Table 2). A good classification performance was also observed in detecting severe SDB [AUC (SE), 0.997 (0.003), $P<0.0001$ ] with the optimal cutoff criterion of CVHR index $\geq 17$, although the performance in detecting mild SDB was modest (Table 2 and Fig. 3).
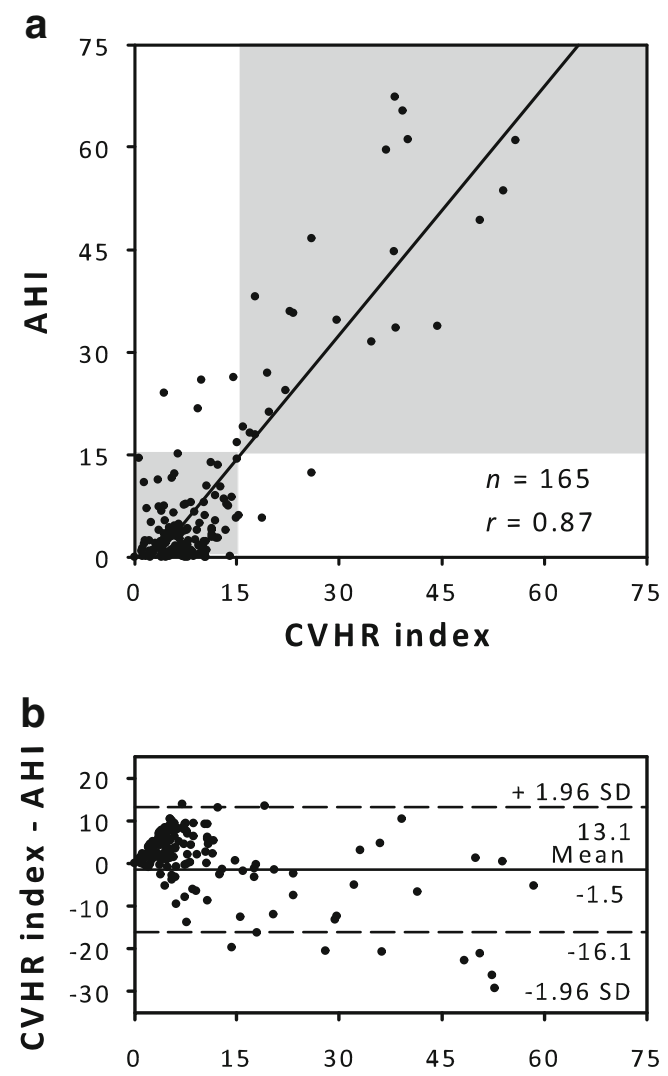

(CVHR index $+A H I) / 2$

Fig. 2 Scatter graphs with the regression line (a) and Bland and Altman plot (b) for the relationships between the CVHR index and apnea-hypopnea index $(A H I)$. In (b), the horizontal solid line and dashed lines indicate the mean difference and the upper and lower limits of agreement (mean $\pm 1.96 \mathrm{SD}$ ), respectively 
Table 2 Classification performance of the CVHR index for identifying patients with different severity of SDB

\begin{tabular}{|c|c|c|c|c|c|c|c|c|c|}
\hline & $n$ & ROC curve AUC (SE) & CVHR index criterion & Sensitivity & Specificity & PPV & NPV & $\mathrm{LR}+$ & $\mathrm{LR}-$ \\
\hline $\mathrm{AHI} \geq 5$ & 62 & $0.796(0.0414)^{* a}$ & $\geq 12^{\mathrm{c}}$ & $60 \%$ & $96 \%$ & $90 \%$ & $80 \%$ & 15.4 & 0.42 \\
\hline \multirow[t]{2}{*}{$\mathrm{AHI} \geq 15$} & 26 & $0.974(0.0142)^{*}$ & $\geq 14^{\mathrm{c}}$ & $92 \%$ & $96 \%$ & $83 \%$ & $99 \%$ & 25.7 & 0.08 \\
\hline & - & - & $\geq 15^{\mathrm{b}}$ & $88 \%$ & $97 \%$ & $85 \%$ & $98 \%$ & 30.7 & 0.12 \\
\hline $\mathrm{AHI} \geq 30$ & 16 & $0.997(0.003)^{*}$ & $\geq 17^{\mathrm{c}}$ & $100 \%$ & $96 \%$ & $72 \%$ & $100 \%$ & 24.8 & 0.00 \\
\hline
\end{tabular}

$A U C$ area under the curve, $C V H R$ cyclic variation of heart rate, $L R+$ likelihood ratio for positive test, $L R$ - likelihood ratio for negative test, $N P A$ negative predictive value, $P P V$ positive predictive value, $R O C$ receiver operating characteristic

$* P<0.0001$ (against $\mathrm{AUC}=0.5$ )

${ }^{a}$ Significantly lower than AUCs for AHI $\geq 15$ and AHI $\geq 30$

${ }^{\mathrm{b}}$ Predetermined criterion of CVHR index for detecting moderate-to-severe SDB [19]

${ }^{\mathrm{c}}$ Cutoff threshold determined in the present study as the value corresponding to the highest average of sensitivity and specificity

Table 3 shows the results of interval LR analysis. There was a very large reduction in the probability of severe SDB $(\mathrm{AHI} \geq 30$ ), when CVHR index was $<15$; a very large increase in the probability of SDB (AHI $\geq 5)$ and a large increase in that of moderate-to-severe SDB (AHI $\geq 15$ ), when CVHR index was $\geq 15$; and a very large increase in the probability of moderate-to-severe $\mathrm{SDB}(\mathrm{AHI} \geq 30$ ), when CVHR index was $\geq 30$.

Age, comorbidities, and autonomic function had no substantial impact on the classification performance in detecting moderate-to-severe SDB by the criterion of CVHR index $\geq 15$ (Table 4). The AUC of the ROC curve did not differ significantly between subjects grouped by older age ( $\geq 52$ years, the 75 th percentile value) or by the presence of obesity (BMI $\geq 25 \mathrm{~kg} / \mathrm{m}^{2}$ ), hypertension, diabetes mellitus, dyslipidemia, and decreased cardiac autonomic function.

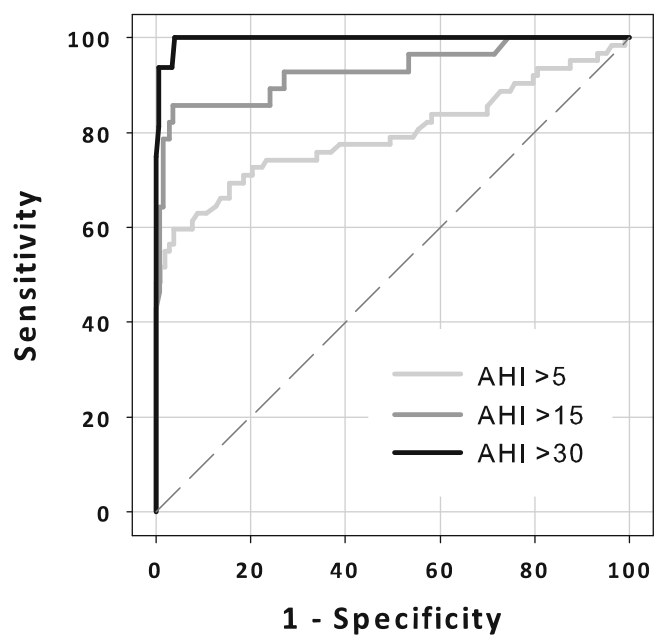

Fig. 3 Receiver operating characteristic (ROC) curves of the CVHR index to identify patients with different severity of SDB

\section{Discussion}

This is the first population-based study to report the diagnostic accuracy of automated ECG-based detection of SDB. In a survey of all male workers of a transport company, the ACAT algorithm identified moderate-to-severe SDB patients with $88 \%$ sensitivity and $97 \%$ specificity with a predetermined criterion of CVHR index $\geq 15$. Also, interval LR analysis revealed that CVHR index $<15$ is a useful criterion for reducing the probability of severe SDB (AHI $\geq 30$ ), CVHR index $\geq 15$ is for increasing the probability of SDB (AHI $\geq 5$ ), and CVHR index $\geq 30$ is for increasing the probability of moderate-to-severe $\mathrm{SDB}(\mathrm{AHI} \geq 15)$. The accuracy was retained in the subgroups divided by age, obesity, hypertension, diabetes mellitus, dyslipidemia, and decreased autonomic function. These observations indicate that the automated ECG-based detection of CVHR during sleep may be used as a screening tool for moderate-to-severe SDB among male workers.

This study has several strengths as a diagnostic study [28]. First, we could performed a survey of all male workers in a company and obtain both the index test (CVHR index) and the reference standard (AHI by a standard overnight attended polysomnography) from all subjects. The observations were of complete samples from a population of male workers. Second, because both CVHR index and AHI were evaluated simultaneously, there was neither time lag nor treatment effects between the index test and the reference standard. Third, the majority of the subjects consisted of long-distance truck drivers, a representative population in whom the screening for SDB is of particular importance [12]. Finally, we estimated the variability of diagnostic accuracy between subgroups divided by age, obesity, and the diseases commonly observed among SDB patients [3].

The ACAT algorithm may perform excellently in comparison to other ECG-based algorithms for SDB detection. 
Table 3 Interval likelihood ratio of the CVHR index for identifying patients with different severity of SDB

\begin{tabular}{llllll}
\hline & \multicolumn{2}{l}{ CVHR index } & & Pretest probability \\
\cline { 2 - 4 } & $<10(n=110)$ & $10-15(n=29)$ & $15-30(n=15)$ & $\geq 30(n=11)$ \\
\hline $\mathrm{AHI} \geq 5$ & $0.39(19 \%)$ & $1.78(52 \%)$ & $\infty(100 \%)$ & $\infty(100 \%)$ & $38 \%$ \\
$\mathrm{AHI} \geq 15$ & $0.10(2 \%)$ & $0.40(7 \%)$ & $14.7(73 \%)$ & $\infty(100 \%)$ & $16 \%$ \\
$\mathrm{AHI} \geq 30$ & $0.00(0 \%)$ & $0.00(0 \%)$ & $4.66(33 \%)$ & $\infty(100 \%)$ & $10 \%$ \\
\hline
\end{tabular}

Data are LR (post-test probability, \%)

$C V H R$ cyclic variation of heart rate, $L R$ likelihood ratio

Several earlier studies [15-17] have used the Physionet Sleep Apnea-ECG database (http://www.physionet.org/ physiobank/database/apnea-ecg/) to examine the performance of the algorithms and reported an accuracy of 90 $100 \%$. This database consists of 70 ECG samples including 42 patients with $\mathrm{AHI} \geq 15$ and 23 normal subjects $(\mathrm{AHI}<5)$. The ACAT algorithm also showed a good performance [AUC, 0.979 (95\% CI, 0.912-0.998); $90 \%$ sensitivity and $100 \%$ specificity] for this database [19]. Although only a few studies have examined the performance of the algorithms in clinical settings, they have reported modest diagnostic accuracies [18, 20,29]. In a study of 150 patients referred to a university hospital for clinically suspected SDB, Roche et al. [18] reported that an algorithm that used the relative power of the very low frequency component detected patients with AHI $\geq 15$ with an AUC of 0.70 , a sensitivity of $64 \%$, and a specificity of $69 \%$. The ACAT algorithm, however, has been reported to detect patients with $\mathrm{AHI} \geq 15$ with an AUC of 0.913 , a sensitivity of $83 \%$, and specificity of $88 \%$ in a study of 887 consecutive patients referred for diagnostic polysomnography [19].

The advantage of the ACAT algorithm may be derived from its feature of an adaptive threshold. By visual inspection of R-R intervals, Guilleminault et al. [14] reported that the CVHR was not observed in patients with denervated transplanted heart or with severe autonomic neuropathy and that it was blunted in those with moderate autonomic neuropathy and with Shy-Drager syndrome. They also observed in subjects with normal autonomic function that the bradycardic component of the CVHR showed a progressive reduction with increasing doses of intravenous atropine. Cardiac vagal activity decreases with aging [30] and is reduced in patients with diabetes mellitus [31] and dyslipidemia [32]. These conditions are commonly associated with SDB $[4,8,33]$ and have been reported to increase the failure of CVHR detection [22]. The adaptive threshold of the ACAT algorithm may help maintain the good classification performance even in these subjects.

Table 4 Effects of age and comorbidities on the classification performance of the CVHR index (cutoff, $\geq 15$ )

\begin{tabular}{|c|c|c|c|c|c|c|c|c|c|}
\hline Subgroup & $n$ & $\mathrm{AHI} \geq 15 n(\%)$ & ROC curve AUC (SE) & Sensitivity & Specificity & PPV & NPV & $\mathrm{LR}+$ & LR- \\
\hline Age $<52$ years & 124 & $12(10 \%)$ & $0.963(0.0225)^{*}$ & $83 \%$ & $97 \%$ & $77 \%$ & $98 \%$ & 31.1 & 0.17 \\
\hline Age $\geq 52$ years & 41 & $14(34 \%)$ & $0.995(0.0064)^{*}$ & $93 \%$ & $93 \%$ & $93 \%$ & $93 \%$ & 12.5 & 0.08 \\
\hline $\mathrm{BMI}<25 \mathrm{~kg} / \mathrm{m}^{2}$ & 115 & $9(8 \%)$ & $0.968(0.0266)^{*}$ & $89 \%$ & $98 \%$ & $80 \%$ & $99 \%$ & 47.1 & 0.11 \\
\hline $\mathrm{BMI} \geq 25 \mathrm{~kg} / \mathrm{m}^{2}$ & 50 & $17(34 \%)$ & $0.970(0.0223)^{*}$ & $898 \%$ & $97 \%$ & $94 \%$ & $94 \%$ & 29.1 & 0.12 \\
\hline Hypertension (-) & 108 & $7(6 \%)$ & $0.996(0.0040)^{*}$ & $100 \%$ & $99 \%$ & $88 \%$ & $100 \%$ & 101.0 & 0.00 \\
\hline Hypertension $(+)$ & 57 & $19(33 \%)$ & $0.939(0.0369)^{*}$ & $89 \%$ & $95 \%$ & $88 \%$ & $93 \%$ & 167.0 & 0.11 \\
\hline Diabetes $(-)$ & 152 & $20(13 \%)$ & $0.952(0.0567)^{*}$ & $95 \%$ & $96 \%$ & $79 \%$ & $99 \%$ & 25.8 & 0.05 \\
\hline Diabetes $(+)$ & 13 & $6(46 \%)$ & $0.952(0.0567)^{*}$ & $83 \%$ & $100 \%$ & $100 \%$ & $88 \%$ & $\infty$ & 0.17 \\
\hline Dyslipidemia (-) & 82 & $5(6 \%)$ & $0.990(0.0095)^{*}$ & $80 \%$ & $99 \%$ & $80 \%$ & $99 \%$ & 61.6 & 0.20 \\
\hline Dyslipidemia $(+)$ & 83 & $21(25 \%)$ & $0.970(0.0191)^{*}$ & $90 \%$ & $97 \%$ & $91 \%$ & $97 \%$ & 28.0 & 0.10 \\
\hline $\mathrm{SDNN} \geq 75 \mathrm{~ms}$ & 139 & $20(14 \%)$ & $0.982(0.0144)^{*}$ & $95 \%$ & $97 \%$ & $83 \%$ & $99 \%$ & 28.3 & 0.05 \\
\hline $\mathrm{SDNN}<75 \mathrm{~ms}$ & 26 & $6(23 \%)$ & $0.958(0.0368)^{*}$ & $83 \%$ & $95 \%$ & $83 \%$ & $95 \%$ & 16.7 & 0.18 \\
\hline
\end{tabular}

Abbreviations are explained in the footnote to Table 2

$* P<0.0001$ (against $\mathrm{AUC}=0.5$ ) 
The ACAT algorithm requires only $\mathrm{R}-\mathrm{R}$ interval time series data. The most promising application of this algorithm seems to be screening for sleep apnea by Holter ECG. According to the Survey of Medical Care Activities in Public Health Insurance conducted by the Japanese Ministry of Health, Labor and Welfare in 2008, at least 1,270,000 Holter ECG examinations are performed each year in Japan alone. Holter ECG is used as a routine examination in most clinical facilities; in many of them, digitized Holter ECG data are stored in re-analyzable forms. Also, Holter ECG scanners used for analyzing recorded ECG signals unexceptionally measure beat-to-beat R-R intervals as a fundamental function. Thus, the data that are necessary for the ACAT algorithm can be obtained at small additional cost. Furthermore, the cardiovascular patients, the most likely subjects of Holter ECG examination, are also the high-risk population of SDB [3]. The installation of ACAT algorithm into Holter ECG scanners may provide useful screening tool for sleep apnea in these patients.

The present study has several limitations. First, the studied population consisted of only male workers. Thus, the performance of the ACAT algorithm in other populations is unclear, although our previous observation in subjects referred for polysomnography indicated that the classification performance was maintained even in 155 female subjects and in 145 subjects aged $\geq 65$ years [19]. Second, we studied the ECG signal extracted from polysomnographic recordings obtained in a laboratory. The performance may be affected by the recording environment. It is not clear if our findings can be directly extended to ambulatory ECG recorded during daily life. Third, we used TIB as the denominator for calculating CVHR index, while we used TST as the denominator for AHI. This might have affected the association between CVHR and AHI. Their correlation coefficients were comparable, however, even when TIB was used for calculating AHI ( $r=0.87$ vs. 0.86). Fourth, we did not evaluate the effects of medications because we were unable to have consent from the subjects to access their clinical medical recordings. We performed, however, a full health checkup of the subjects and observed that the classification performance was retained even in the subjects with hypertension, diabetes mellitus, dyslipidemia, and decreased autonomic function. Fifth, it should be noted that the CVHR index may not be used for estimating AHI. Although the CVHR index correlated with AHI, the Bland and Altman plot showed increasing disagreement with increasing AHI. Also, the optimal cutoff criterion of CVHR index for identifying patients with an $\mathrm{AHI} \geq 30$ was $\geq 17$. These results suggest that the CVHR index, if used as a quantitative index, would underestimate the AHI in patients with severe SDB. The CVHR index should not be used for AHI estimation; it should be used only for per-subject screening tool for moderate-to-severe SDB. Finally, to confirm the cost- effectiveness of ECG screening for sleep apnea, comparisons with other portable monitors such as those with oximetry are necessary in future studies.

\section{Conclusions}

We observed that analysis of CVHR from ECG by the automated algorithm of ACAT identified patients with moderate-to-severe SDB in a survey of all male workers in a transport company. Our observations suggest that automated ECG detection of CVHR may be a useful screening tool for moderate-to-severe SDB among male workers.

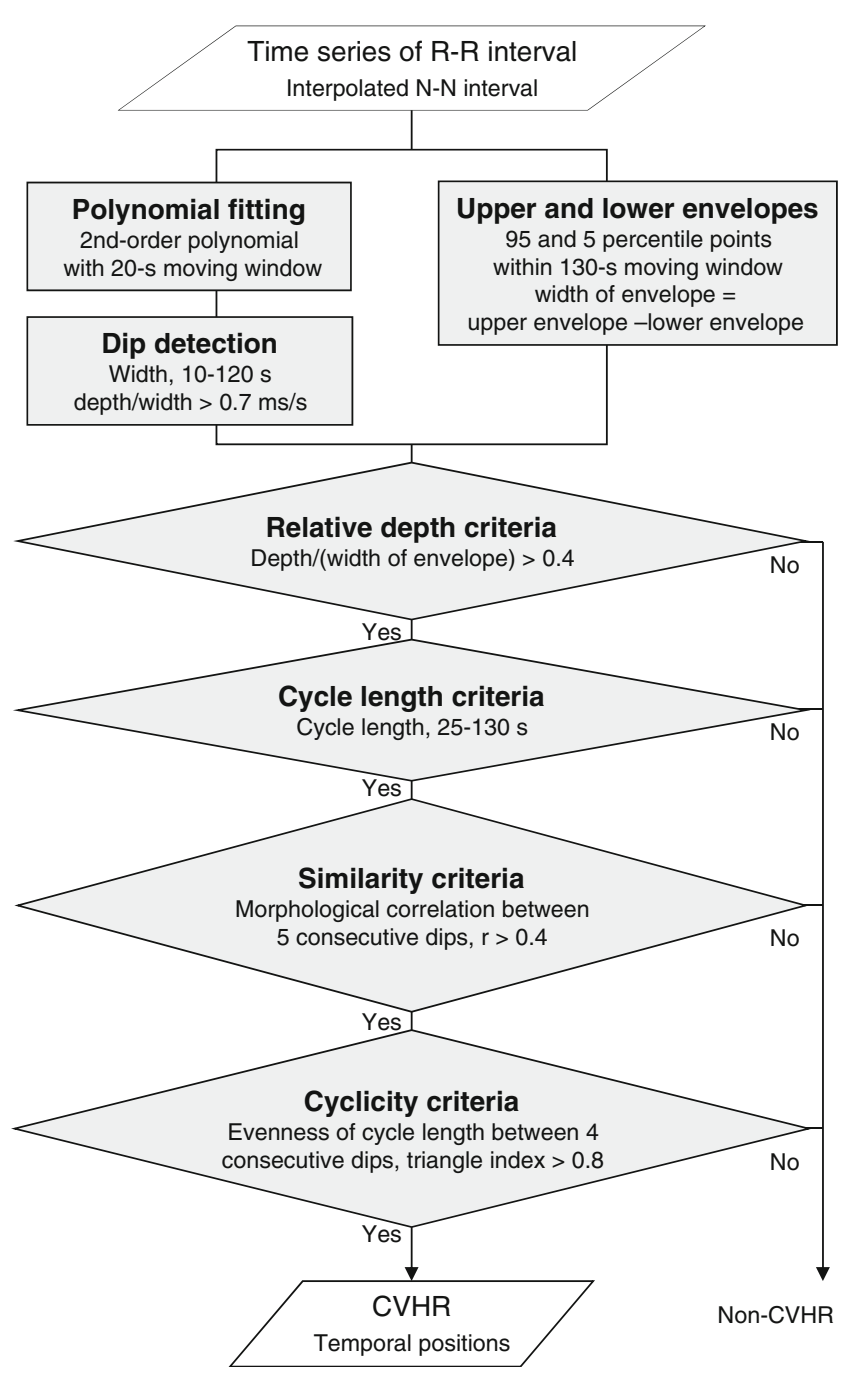

Fig. 4 Algorithm of autocorrelated wave detection with adaptive threshold (ACAT). The algorithm detects the temporal positions of cyclic variation of heart rate (CVHR) in the interbeat interval time series as the cyclic and autocorrelated dips that meet four specific criteria (modified from Fig. 1 in Ref. [19]) 
Acknowledgments This work was supported by the Japan Society for the Promotion of Science, Japan [Grant-in-Aid for Scientific Research (C) 18590552 (to Dr. Nomiyama), 20590832, 23591055 (to Dr. Hayano)] and by the Ministry of Health, Labour and Welfare, Japan [Grant for the Respiratory Failure Research Group (to Dr. Fujimoto), Research Grant for Nervous and Mental Disorders 20B-7, 23-2 (to Dr. Hayano)].

Conflict of interest The algorithm of autocorrelated wave detection with adaptive threshold (ACAT) is installed in a Holter ECG scanner (Cardy Analyzer, Suzuken Company Limited, Nagoya, Japan). Mr. Kawai is an employee of Suzuken Company Limited. Dr. Kodama is an advisor and Dr. Hayano is a consultant of Suzuken Company Limited. Other authors have reported that no conflicts of interest exist with any companies/organizations whose products or services may be discussed in this article.

Open Access This article is distributed under the terms of the Creative Commons Attribution License which permits any use, distribution, and reproduction in any medium, provided the original author(s) and the source are credited.

\section{Appendix}

Automated algorithm of autocorrelated wave detection with adaptive threshold (ACAT) for detecting cyclic variation of heart rate (CVHR) [19]

Fig. 5 Schema showing the processes for detecting CVHR by the ACAT algorithm. a Original $\mathrm{R}-\mathrm{R}$ interval (thin solid line), second-order polynomial fitting line (heavy solid line), and the upper and lower envelopes of the fitting line (dashed lines). b The depth of dips relative to the instantaneous width of envelopes. c The morphological comparisons of each dip (shaded box) with the two preceding and two subsequent dips (open boxes). d Mean morphological correlation coefficients. e Temporal positions of dips detected as CVHR
The ACAT algorithm is a time-domain method that uses only interbeat interval data. The algorithm detects the CVHR as cyclic and autocorrelated dips in interbeat interval time series and determines the temporal position of the individual dips comprising the CVHR (Figs. 4 and 5). The processes of the ACAT algorithm were as follows: Interbeat interval time series were smoothed by second-order polynomial fitting, and all dips in the smoothed trend with widths between 10 and $120 \mathrm{~s}$ and depth-to-width ratios of $>0.7 \mathrm{~ms} / \mathrm{s}$ were detected. Also, the upper and lower envelopes of the interbeat interval variations were calculated as the 95th and 5 th percentile points, respectively, within a sifting window with a width of $130 \mathrm{~s}$. Then, the dips that met the following criteria were considered CVHR: (1) a relative dip depth $>40 \%$ of the envelope range at that point (adaptive threshold), (2) interdip intervals (cycle length) between 25 and $130 \mathrm{~s}$, (3) a waveform similar to those of the two preceding and two subsequent dips with a mean morphological correlation coefficients $>0.4$ (autocorrelated wave), and (4) three cycle lengths between four consecutive dips that meet the following equivalence criteria:

$$
\left(3-2 l_{1} / s\right)\left(3-2 l_{2} / s\right)\left(3-2 l_{3} / s\right)>0.8,
$$
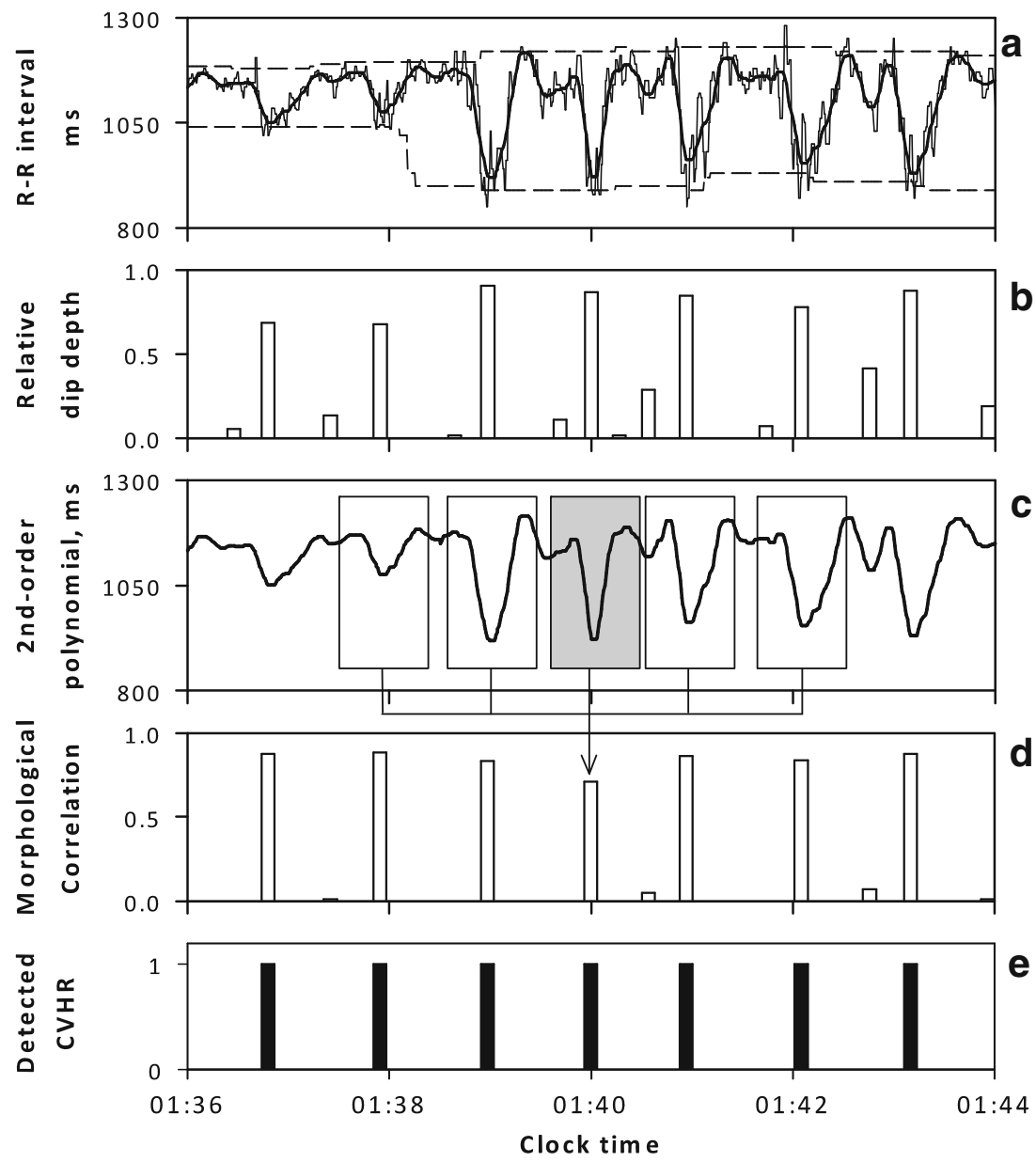
where $l_{1}, l_{2}$, and $l_{3}$ are three consecutive cycle lengths and $s=\left(l_{1}+l_{2}+l_{3}\right) / 3$. The number of dips comprising the CVHR is counted and the mean number of the dips per hour of time in bed is calculated as the CVHR index.

\section{References}

1. Young T, Finn L, Peppard PE, Szklo-Coxe M, Austin D, Nieto FJ, Stubbs R, Hla KM (2008) Sleep disordered breathing and mortality: eighteen-year follow-up of the Wisconsin sleep cohort. Sleep 31:1071-1078

2. Punjabi NM (2008) The epidemiology of adult obstructive sleep apnea. Proc Am Thorac Soc 5:136-143

3. Somers VK, White DP, Amin R, Abraham WT, Costa F, Culebras A, Daniels S, Floras JS, Hunt CE, Olson LJ, Pickering TG, Russell R, Woo M, Young T (2008) Sleep apnea and cardiovascular disease: an American Heart Association/American College of Cardiology Foundation Scientific Statement from the American Heart Association Council for High Blood Pressure Research Professional Education Committee, Council on Clinical Cardiology, Stroke Council, and Council On Cardiovascular Nursing. In collaboration with the National Heart, Lung, and Blood Institute National Center on Sleep Disorders Research (National Institutes of Health). Circulation 118:1080-1111

4. Peppard PE, Young T, Palta M, Skatrud J (2000) Prospective study of the association between sleep-disordered breathing and hypertension. N Engl J Med 342:1378-1384

5. Peker Y, Carlson J, Hedner J (2006) Increased incidence of coronary artery disease in sleep apnoea: a long-term follow-up. Eur Respir J 28:596-602

6. Shamsuzzaman AS, Gersh BJ, Somers VK (2003) Obstructive sleep apnea: implications for cardiac and vascular disease. JAMA 290:1906-1914

7. Arzt M, Young T, Finn L, Skatrud JB, Bradley TD (2005) Association of sleep-disordered breathing and the occurrence of stroke. Am J Respir Crit Care Med 172:1447-1451

8. Punjabi NM, Shahar E, Redline S, Gottlieb DJ, Givelber R, Resnick HE (2004) Sleep-disordered breathing, glucose intolerance, and insulin resistance: the Sleep Heart Health Study. Am J Epidemiol 160:521-530

9. Sim JJ, Rasgon SA, Kujubu DA, Kumar VA, Liu IL, Shi JM, Pham TT, Derose SF (2009) Sleep apnea in early and advanced chronic kidney disease: Kaiser Permanente Southern California cohort. Chest 135:710-716

10. Peppard PE, Szklo-Coxe M, Hla KM, Young T (2006) Longitudinal association of sleep-related breathing disorder and depression. Arch Intern Med 166:1709-1715

11. Young T, Peppard PE, Gottlieb DJ (2002) Epidemiology of obstructive sleep apnea: a population health perspective. Am J Respir Crit Care Med 165:1217-1239

12. Tregear S, Reston J, Schoelles K, Phillips B (2009) Obstructive sleep apnea and risk of motor vehicle crash: systematic review and meta-analysis. J Clin Sleep Med 5:573-581

13. Young T, Evans L, Finn L, Palta M (1997) Estimation of the clinically diagnosed proportion of sleep apnea syndrome in middle-aged men and women. Sleep 20:705-706

14. Guilleminault C, Connolly S, Winkle R, Melvin K, Tilkian A (1984) Cyclical variation of the heart rate in sleep apnoea syndrome. Mechanisms, and usefulness of $24 \mathrm{~h}$ electrocardiography as a screening technique. Lancet 1:126-131

15. Penzel T, McNames J, Murray A, de Chazal P, Moody G, Raymond B (2002) Systematic comparison of different algorithms for apnoea detection based on electrocardiogram recordings. Med Biol Eng Comput 40:402-407

16. Khandoker AH, Palaniswami M, Karmakar CK (2009) Support vector machines for automated recognition of obstructive sleep apnea syndrome from ECG recordings. IEEE Trans Inf Technol Biomed 13:37-48

17. Mendez MO, Corthout J, Van Huffel S, Matteucci M, Penzel T, Cerutti S, Bianchi AM (2010) Automatic screening of obstructive sleep apnea from the ECG based on empirical mode decomposition and wavelet analysis. Physiol Meas 31:273-289

18. Roche F, Celle S, Pichot V, Barthelemy JC, Sforza E (2007) Analysis of the interbeat interval increment to detect obstructive sleep apnoea/hypopnoea. Eur Respir J 29:1206-1211

19. Hayano J, Watanabe E, Saito Y, Sasaki F, Fujimoto K, Nomiyama T, Kawai K, Kodama I, Sakakibara H (2011) Screening for obstructive sleep apnea by cyclic variation of heart rate. Circ Arrhythm Electrophysiol 4:64-72

20. Poupard L, Court-Fortune I, Pichot V, Chouchou F, Barthelemy JC, Roche F (2011) Use of high-frequency peak in spectral analysis of heart rate increment to improve screening of obstructive sleep apnoea. Sleep Breath 15:837-843

21. Lado MJ, Vila XA, Rodríguez-Liñares L, Méndez AJ, Olivieri DN, Félix P (2009) Detecting sleep apnea by heart rate variability analysis: assessing the validity of databases and algorithms. J Med Syst 35:473-481

22. Stein PK, Duntley SP, Domitrovich PP, Nishith P, Carney RM (2003) A simple method to identify sleep apnea using Holter recordings. J Cardiovasc Electrophysiol 14:467-473

23. Agatsuma T, Fujimoto K, Komatsu Y, Urushihata K, Honda T, Tsukahara T, Nomiyama T (2009) A novel device (SD-101) with high accuracy for screening sleep apnoea-hypopnoea syndrome. Respirology 14:1143-1150

24. The AASM Manual for the Scoring of Sleep and Associated Events: Rules, Terminology and Technical Specifications. 1st ed. American Academy of Sleep Medicine, Westchester; 2007.

25. Bland JM, Altman DG (1986) Statistical methods for assessing agreement between two methods of clinical measurement. Lancet $1: 307-310$

26. Hanley JA, McNeil BJ (1983) A method of comparing the areas under receiver operating characteristic curves derived from the same cases. Radiology 148:839-843

27. Flemons WW, Littner MR (2003) Measuring agreement between diagnostic devices. Chest 124:1535-1542

28. Bossuyt PM, Reitsma JB, Bruns DE, Gatsonis CA, Glasziou PP, Irwig LM, Lijmer JG, Moher D, Rennie D, de Vet HC (2003) Towards complete and accurate reporting of studies of diagnostic accuracy: the STARD Initiative. Ann Intern Med 138:40 44

29. Zamarron C, Hornero R, del Campo F, Abasolo D, Alvarez D (2006) Heart rate regularity analysis obtained from pulse oximetric recordings in the diagnosis of obstructive sleep apnea. Sleep Breath 10:83-89

30. Shannon DC, Carley DW, Benson H (1987) Aging of modulation of heart rate. Am J Physiol 253:H874-H877

31. Boulton AJ, Vinik AI, Arezzo JC, Bril V, Feldman EL, Freeman R, Malik RA, Maser RE, Sosenko JM, Ziegler D (2005) Diabetic neuropathies: a statement by the American Diabetes Association. Diabetes Care 28:956-962

32. Pehlivanidis AN, Athyros VG, Demitriadis DS, Papageorgiou AA, Bouloukos VJ, Kontopoulos AG (2001) Heart rate variability after long-term treatment with atorvastatin in hypercholesterolaemic patients with or without coronary artery disease. Atherosclerosis 157:463-469

33. Peppard PE, Young T, Palta M, Dempsey J, Skatrud J (2000) Longitudinal study of moderate weight change and sleepdisordered breathing. JAMA 284:3015-3021 